\title{
Prueba de comprensión lectora en gallego para escolares de segundo de primaria
}

\author{
Test of reading comprehension in Galician for students of second grade
}

\author{
Paula Outón Oviedo* \\ *Universidad de Santiago de Compostela
}

\begin{abstract}
Resumen
En este trabajo presentamos una prueba de cribado para averiguar qué escolares de segundo de Educación Primaria tienen dificultades en comprensión lectora en gallego (COLEGA2) y necesitan una atención especial. Dicha prueba forma parte de una batería de instrumentos más amplia, en castellano y gallego, que tiene como objetivo evaluar el rendimiento en lectura y escritura al inicio de ese curso académico. Se describe el marco teórico de partida, el proceso seguido para la elaboración de la misma, sus principales características y algunos resultados obtenidos. La aplicación de COLEGA2 puede ofrecernos información sobre los niveles de comprensión en nuestra comunidad.

Palabras clave: comprensión lectora en gallego, evaluación de la comprensión lectora en gallego, dificultades en comprensión lectora.
\end{abstract}

\begin{abstract}
In this paper we sense beforehand a test of screening to quarrel what students of the second course of Primary Education have difficulties in reading comprehension in Galician (COLEGA2) and need a special attention. This test is part of a broader set of instruments, in Castilian and Galician, which aims to assess performance in reading and writing at the beginning of this academic course. There is described the theoretical framework of test, the process followed for the production of the same, its main characteristics and some results obtained. The application of COLEGA2 can offer information about the levels of reading comprehension in our community.

Keywords: reading comprehension in Galician, reading comprehension assessment in Galician, difficulties in reading comprehension.
\end{abstract}

Leer es una actividad humana muy compleja, por lo que es lógico que no exista un modelo explicativo único, universalmente aceptado (Ruddell, Ruddell \& Singer, 1994; Britton \& Graesser, 1996; Ruddell \& Unrau, 2004). Esta complejidad tiene que ver con el hecho de que leemos una variedad enorme de textos sobre los más diversos temas (cortos-extensos, literarios-informativos, bien-mal escritos...); con que leemos con propósitos predominantes muy diferentes (por placer, para informarnos, para saber cómo realizar determinadas actividades, para clarificar nuestro pensamiento...); con que leemos en situaciones y contextos muy diferentes (privados, públicos, escolares, laborales...) y, muy fundamentalmente, tiene que ver con una serie de características inherentes al lenguaje verbal (arbitrariedad, linealidad, generalidad, versatilidad...). Esta complejidad del proceso la afronta el lector desplegando varios subprocesos, que hemos procurado tener en cuenta al elaborar la prueba que presentamos en este trabajo. Pero hay que ser conscientes de que es imposible hacer justicia cabal a la complejidad de la comprensión de textos en una prueba administrada en un período de tiempo corto (OECD, 2001).

Comprender significa abarcar el todo. No se comprende el significado del texto si no se construye un modelo de la situación o modelo referencial, esto es, si de algún modo no reconstruimos, a partir del texto, la realidad extralingüística o referente (Díaz \& De Vega, 2003). No basta, pues, ni con una comprensión a nivel proposicional (de significado), ni basta —con más razón-, con una a nivel superficial o verbatim. La tarea no es fácil, porque hay que sobreponerse, por un lado, a la linealidad/secuencialidad inherente al lenguaje verbal $\mathrm{y}$, por otro, hay que rellenar con significados particulares los signos lingüísticos (palabras, oraciones, texto...), que se caracterizan por su generalidad (Vygotsky, 1962). Kintsch (1998) refleja el carácter unitario y la dificultad de la comprensión lectora con estas palabras:

La comprensión implica la formación de todos coherentes con cualidades de tipo guestáltico, a partir de elementos perceptivos y conceptuales básicos. Es un logro extraordinario y maravilloso, ya que hay miles de tales elementos, susceptibles de producir muchas configuraciones diferentes. (p. 93)

No toda la información que contiene un texto figura en él de manera explícita. Últimamente — sobre todo debido a la aportación de la Lingüística pragmática- se ha incidido en el papel que desempeña en la comprensión lectora la generación de inferencias de diverso tipo (locales-globales, necesarias-opcionales, automáticas-conscientes...), que requieren la activación de conocimientos previos, que obviamente no se pueden activar si no se tienen. La cantidad de conocimientos previos, muy relacionada con la riqueza de vocabulario, es un elemento de la comprensión lectora difícil de mejorar, porque requiere su tiempo (Suárez, 1995).

Cuenta con más tradición la preocupación por la diferente importancia de lo que se dice en el texto. Resumir el texto leído, por ejemplo, es una tarea escolar clásica, aunque no haya sido acompañada siempre de una didáctica adecuada. Con frecuencia se asocia con el resumen escrito, lo que es a todas luces muy insatisfactorio: no se puede comprender bien un texto si no se van seleccionando o construyendo las proposiciones más importantes según se va leyendo. Es imprescindible resumir el texto para comprenderlo. Que se nos pida escribir el resumen es una cuestión diferente, $\mathrm{y}$, en todo caso, posterior.

Por lo que respecta a la enorme variedad de textos, clasificables según muy distintos criterios, la dicotomía 
textos literarios vs. textos de información recoge dos finalidades importantes de la lectura: leer para entretenerse y leer para aprender o informarse. Zwann (1996) ha demostrado que estas dos variedades disparan dos maneras distintas de leer, por lo que nos parece que ambas tienen que estar presentes en una prueba de comprensión lectora, aunque sea para escolares de $2^{\circ}$ de Educación Primaria (Wray \& Lewis, 2000). Pero, sin lugar a dudas, la variedad de textos predominante en nuestros días es el texto multimodal, que combina el lenguaje verbal con otros lenguajes (icónico, musical, etc.) para sacar partido de los puntos fuertes de cada uno de ellos.

Por lo que respecta a instrumentos para evaluar la comprensión lectora no contamos con ninguno en gallego, aunque sí en castellano. En esta última lengua tenemos, entre otros: EDIL (González Portal, 1984), TALE (Toro y Cervera, 1984), Pruebas de lectura (de la Cruz, 1988), Pruebas psicopedagógicas de aprendizajes instrumentales (Canals, 1988), Prueba CLP (Alliende, Condemarín \& Milic, 1991), Pruebas ACL (Catalá, Catalá, Molina \& Monclús, 2001), EMLE: TALE-2000 (Toro, Cervera \& Urío, 2002), TIDEL (Secadas, Alfaro \& Cortés, 2003), PROLEC-R (Cuetos, Rodríguez, Ruano \& Arribas, 2007).

El objetivo de este trabajo es presentar una prueba para detectar las dificultades en comprensión lectora en gallego para escolares de segundo curso de Educación Primaria (COLEGA2), respondiendo a una concepción actualizada de comprensión lectora, con especial énfasis en medir lectura "auténtica" de textos. Dicha prueba forma parte de una batería de instrumentos más amplia, en castellano y gallego, que tiene como objetivo evaluar el rendimiento en lectura y escritura al inicio de ese curso académico $^{\mathrm{i}}$.

\section{Sujetos}

\section{Método}

La muestra de tipificación de COLEGA2 estuvo formada por 460 escolares de ambos sexos (un 47,8\% eran niñas y un 52,2\% niños), cuyas edades variaban entre los 6 años y 9 meses y los 7 años y 9 meses. Todos eran alumnos que iniciaban segundo de Educación Primaria en 22 centros educativos de la Comunidad Autónoma de Galicia. Estos alumnos pertenecían a los principales tipos de centros que existen en esta comunidad (público rural, público de villa, público urbano, privado de villa y privado urbano). La Tabla 1 resume las características de la muestra en función del tipo de centro.

Tabla 1.

Muestra de escolares a los que se aplicó COLEGA2

\begin{tabular}{lcccc}
\hline & Rurales & De Villas & Urbanos & Totales \\
\hline Públicos & 115 & 92 & 128 & 335 \\
Privados & 0 & 45 & 80 & 125 \\
Totales & 115 & 137 & 208 & 460 \\
\hline
\end{tabular}

\section{Instrumento}

La prueba está formada por dos textos en gallego, un cuento y un texto informativo, presentados en sendos libros con ilustraciones en color (Qualifications and
Curriculum Authority \& The Stationary Office, 1998). El texto narrativo fue escrito para la prueba por una conocida escritora de literatura infantil, siguiendo unas pautas que se le dieron. El texto informativo fue compuesto por el equipo de investigación, teniendo en cuenta el currículo vigente para el primer ciclo de Educación Primaria del conocimiento del medio. Así pues, a una muestra piloto de escolares que terminaban $1^{\circ}$ de Educación Primaria se les preguntó por escrito qué sabían del tema y qué les gustaría saber. Con esta información se construyó un texto auténticamente informativo, ya que presenta, a la vez, información desconocida y al alcance de los conocimientos e intereses de los escolares. Los textos tienen unas 500 palabras cada uno.

Los alumnos deberán leer cada texto y responder posteriormente a 12 preguntas para cada uno de los textos, conformando la prueba un total de 24 preguntas. Veinte de las mismas son de elección múltiple; las cuatro restantes son de respuesta abierta corta. En cuanto a la profundidad del procesamiento lector, hay ítems de los niveles superficial, proposicional y situacional. En cuanto a la abarcadura o cobertura, hay unos elementos que se refieren a aspectos parciales del texto y otros globales. Se incluyen ítems de distinta importancia. Un número considerable de preguntas exige alguna dosis de inferencia por parte del lector, ya que la respuesta no está explícita en el texto. Finalmente, para contestar a algunas de las preguntas hay que tener en cuenta las ilustraciones. En la Tabla 2 se puede ver el contenido de las preguntas. Se apreciará que los totales no suman 24. Ello es debido a que sólo se recogió en la tabla la caracterización cuando por lo menos dos de los tres jueces que hicieron la evaluación coincidieron en la clasificación. En el caso de “lenguaje”, el total excede 24, porque algunos ítems dependían tanto del lenguaje verbal como del icónico.

Tabla 2

Características de los ítems de COLEGA2

\begin{tabular}{|c|c|c|c|c|c|c|c|c|c|c|c|}
\hline & \multicolumn{3}{|c|}{ Profundidad } & \multicolumn{2}{|c|}{$\begin{array}{c}\text { Abarcadura/ } \\
\text { Cobertura }\end{array}$} & \multicolumn{2}{|c|}{ Importancia } & \multicolumn{2}{|c|}{ Presencia } & \multicolumn{2}{|c|}{ Lenguaje } \\
\hline & Sup. & Pro. & Sit. & Tot. & Parc. & May. & Men. & Exp. & Inf. & Verb. & Icón. \\
\hline Narr. & 4 & 2 & 2 & 2 & 8 & 5 & 4 & 8 & 3 & 10 & 3 \\
\hline Infor. & 5 & 3 & 1 & 3 & 9 & 4 & 8 & 8 & 3 & 9 & 3 \\
\hline Total & 9 & 5 & 3 & 5 & 17 & 9 & 12 & 16 & 6 & 19 & 6 \\
\hline
\end{tabular}

Las preguntas de elección múltiple se puntuaron con 0 ó 1 y las abiertas con $0,0.5$ ó 1, según unas normas elaboradas después de estudiar las respuestas dadas en las aplicaciones experimentales.

\section{Procedimiento}

La prueba fue administrada por tres personas contratadas y entrenadas al efecto, después de algunas aplicaciones piloto a pequeña escala y la aplicación experimental. Este personal contratado tenía el título de maestro/a y el de Pedagogía o Psicopedagogía, además de experiencia docente. La administración de la prueba se llevó a cabo en la primera sesión de la mañana en el mes de octubre, una vez que se recobró el ritmo normal de clase, después de las largas vacaciones estivales. Se trató de presentar a los escolares la prueba como una actividad normal de trabajo del aula. El administrador les pedía que cubriesen, en primer lugar, los datos 
personales (nombre y fecha de nacimiento) en la parte superior de la prueba y luego explicaba la tarea que había que realizar. No se retiraban los textos a la hora de contestar las preguntas. Después de 15 minutos, se recogían las pruebas y se dejaba cinco minutos más a aquellos alumnos que lo solicitasen. En la mayoría de los casos, el profesor del aula optó por estar presente durante la administración, aprovechando el tiempo para cumplimentar una hoja en la que se le pedía la valoración de los alumnos en comprensión lectora y expresión escrita.

\section{Resultados}

En la Tabla 3 se presentan los estadísticos básicos (medias y desviaciones estándares) obtenidos en los dos textos y para el total de la prueba. En estos resultados se puede observar que la comprensión lectora en gallego es bastante insatisfactoria al comienzo de $2^{\circ}$ de Educación Primaria, en especial con los textos informativos, que tanta importancia tienen en la enseñanza-aprendizaje del currículo. Los escolares obtuvieron una media de 12.220 respuestas correctas $(\mathrm{SD}=4.379)$ de un total de 24 preguntas, de las cuales $7.571(\mathrm{SD}=2.829)$ pertenecen al texto narrativo, mientras que $4.649(\mathrm{SD}=2.178)$ se corresponden con el texto informativo.

Tabla3.

Estadísticos básicos de COLEGA2

\begin{tabular}{lccc}
\hline & Total & Narrativo & Informativo \\
\hline Media & 12.220 & 7.571 & 4.649 \\
Desviación típica & 4.379 & 2.829 & 2.178 \\
Alfa & 0.774 & 0.750 & 0.529 \\
\hline
\end{tabular}

En la Figura 1 se muestra una distribución de frecuencias para el total de la prueba. En ella se puede apreciar que un $10 \%$ de los escolares obtuvo 7 puntos o menos en el total de la prueba y que ningún escolar obtuvo la puntuación máxima, 24 puntos.

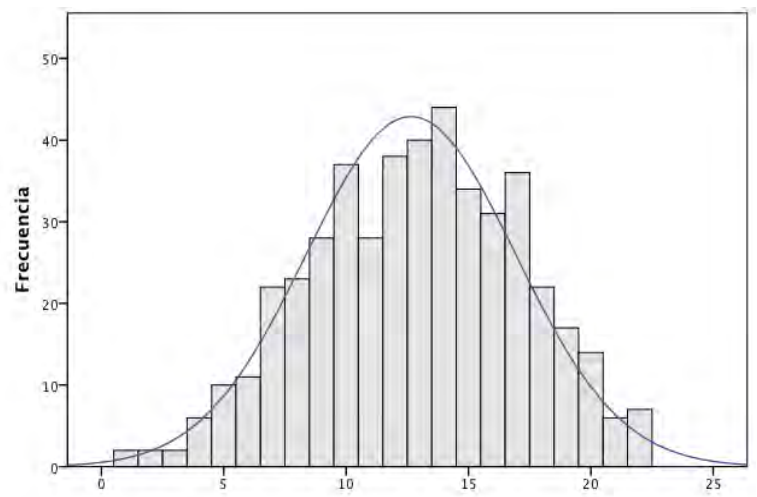

Figura 1. Distribución de frecuencias de GOLEGA2.

Con respecto a la idoneidad de los textos, ítems y alternativas, se analizaron los datos de las respuestas con el programa ITEMAN 3.6. Para el total de la prueba la dificultad de las respuestas correctas está entre 0.20 y 0.87; los índices de discriminación, entre 0.15 y 0.83 ; las correlaciones biseriales puntuales, entre 0.21 y 0.59 . Las correlaciones biseriales puntuales de las alternativas incorrectas son negativas, excepto en 3 ítems (la más alta de estas es de $r_{b p}=0.07$ ). En conjunto consideramos que estas características psicométricas son satisfactorias.

La fiabilidad se estableció a través del coeficiente alfa de Cronbach, obteniéndose un $\alpha$ de 0.774 para el total de la prueba, un $\alpha$ de 0.750 para el texto narrativo y un $\alpha$ de 0.529 para el texto informativo. Para el total de la prueba lo consideramos moderadamente satisfactorio, pero no para cada uno de los textos por separado.

En cuanto a la validez se pidió a los profesores de las aulas en las que se administró el test que valoraran la comprensión lectora de cada alumno utilizando una escala de 1 a 5; siendo 1 equivalente a la calificación de insuficiente, 2 a suficiente, 3 a bien, 4 a notable y 5 a sobresaliente. Posteriormente, se halló la correlación (Tau-c de Kendall) entre las puntuaciones otorgadas por los profesores en esta escala y los resultados en la prueba. El valor de Tau-c de Kendal fue de 0.497. Aunque no fue muy alta, sí resulto ser positiva y significativa al nivel de 0.000 .

\section{Discusión}

Los resultados presentados en la sección anterior aportan información tanto sobre el nivel de comprensión lectora en gallego de los escolares que comienzan $2^{\circ}$ de Educación Primaria en la Comunidad Autónoma de Galicia, como sobre las características psicométricas de la prueba de cribado de comprensión lectora en gallego (COLEGA2). En lo que se refiere al nivel de comprensión de los escolares gallegos, podemos decir que hay bastantes alumnos que no comprenden satisfactoriamente los textos, en particular, los predominantemente informativos. Estos resultados nos hacen pensar en que pueda haber alumnos con dificultades en exactitud y/o automatización lectora y que no puedan dedicar sus recursos atencionales a otros procesos cognitivos superiores, como la comprensión, o alumnos con dificultades en los procesos más relacionados con la comprensión lectora como el desconocimiento del vocabulario, la construcción del significado de las oraciones, la falta o no activación de los conocimientos previos, la no identificación de la información relevante en detrimento de lo que parece ser trivial, etc. Por otra parte, el hecho de que el texto informativo resultase comparativamente más difícil que el narrativo puede deberse a un efecto de la estrategia de aplicación de las pruebas: la del texto informativo siempre se realizó en la segunda sesión, después de haber realizado la correspondiente al texto narrativo. También puede haber influido el hecho de que el texto informativo no responda a una estructura tan canónica como la que tienen las historias. Otra posible razón es que en primero de primaria se suele dar más importancia a los textos narrativos que a los informativos (Wray \& Lewis, 2000).

Con respecto a las características psicométricas señaladas en el apartado anterior, consideramos que la prueba COLEGA2 es un instrumento fiable y válido para 
medir el nivel de comprensión lectora en gallego de los escolares que comienzan $2^{\circ}$ de Educación Primaria. Responde a una concepción de comprensión lectora actualizada, con especial énfasis en medir lectura "auténtica” de textos narrativos e informativos. Además, se trata —que sepamos- de la primera prueba en compresión lectora en gallego.

Sin embargo, creemos que hay que constatar los resultados obtenidos en COLEGA2 con el juicio que tiene el profesor acerca de la comprensión lectora del escolar, ya que este tiene una evidencia durante un lapso de tiempo mucho mayor y procedente de situaciones de lectura mucho más variadas. Si coinciden ambos juicios, se actúa consecuentemente: si el escolar tiene dificultades, se procederá a una intervención intensiva e individualizada durante el curso. Si no coinciden los resultados en COLEGA2 con el juicio del profesor, hay que abordar el conflicto y resolverlo.

Esperamos que la utilización de COLEGA2 por parte de los orientadores de los centros puede confirmar la utilidad de la prueba, aporte más información sobre los niveles de comprensión lectora en gallego, sugiera mejoras en la prueba y, en definitiva, sirva de palanca para elevar el nivel de comprensión lectora de los escolares gallegos.

\section{Referencias}

Alliende, F., Condemarín, M. \& Milicic, N. (1991). CLP. Prueba de Comprensión Lectora de Complejidad Lingüística Progresiva. Madrid: CEPE.

Britton, B. K. \& Graesser, A. C. (1996). Models of Understanding Text. Mahwah, N. J.: L.E.A.

Canals, R. (1988). Pruebas psicopedagógicas de aprendizajes instrumentales. Ciclos inicial y medio de E.G.B. Barcelona: Onda.

Catalá, G., Catalá, M., Molina, E. \& Monclús, R. (2001). Evaluación de la comprensión lectora. Pruebas ACL (1 ${ }^{\circ}$ $6^{\circ}$ de Primaria). Barcelona: Graó.

Cuetos, F., Rodríguez, B., Ruano, E. \& Arribas, D. (2007). PROLEC-R. Batería de Evaluación de los Procesos Lectores, Revisada. Madrid: TEA.

De la Cruz, Mª V. (1988). Pruebas de lectura. Niveles 1, 2. Madrid: TEA.

Díaz, M. \& De Vega, M. (2003). Modelos de situación e inferencias en la comprensión de textos. En J. A. León (Ed.), Conocimiento y discurso. Claves para inferir y comprender (pp. 139-152). Madrid: Pirámide.

González Portal, M. D. (1989). EDIL-1. Exploración de las dificultades individuales de lectura. Madrid: TEA.

Kintsch, W. (1998). Comprehension: a paradigm for cognition. Cambridge: C.U.P.

OECD (2001). Knowledge and skills for Life. First results from PISA 2000. París: OECD.

Qualifications and Curriculum Authority \& The Stationary Office (1998). Official National Test Papers. Key Stage 1 English and Maths Tests. London: The Stationery Office.

Ruddell, R. B. \& Unrau, N. J. (2004). Theoretical Models and Processes of Reading. Newark, DE: I.R.A.

Ruddell, R. B., Ruddell, M. R. \& Singer, H. (1994). Theoretical Models and Processes of Reading. Newark, Delaware: I.R.A.
Secadas, F., Alfaro, I. J. \& Cortés, J. (2003). Leer es fácil. Fundamentos psicopedagógicos del aprendizaje de la lectura. Madrid: CEPE.

Suárez, A. (1995). La comprensión lectora desde una perspectiva pragmático comunicativa. Innovación Educativa, 5, 207-224.

Toro, J. \& Cervera, M. (1984). TALE. Test de Análisis de Lectoescritura. Madrid: Visor.

Toro, J., Cervera, M. \& Urío, C. (2002). Escalas Magallanes de Lectura y Escritura. EMLE-TALE 2000. Burceña-Barakaldo: Albor-Cohs.

Vygotsky, L. S. (1962). Thought and language. Cambridge, MA, US: MIT Press.

Wray, D. \& Lewis, M. (2000). Aprender a leer y escribir textos de información. Madrid: Morata.

Zwaan, R.A. (1996). Toward a Model of Literary Comprehension. En B. K. Britton \& A. C. Graesser (Eds). Models of understanding text (pp. 241-255). Mahwah, N.J.: L.E.A.

i Proyecto de investigación PGIDT/PGIDIT (07SEC015214PR) Batería de evaluación de la competencia en lenguaje escrito en gallego y castellano, subvencionado por la Xunta de Galicia y fondos FEDER 